\title{
A pilot study of recovery room bypass ("fast-track protocol") in a community hospital
}

\author{
[Éviter la salle de réveil, l'étude pilote d'un "protocole accéléré" dans un hôpital \\ communautaire]
}

Peter G. Duncan MD FRCPC, ${ }^{*}$ John Shandro MD FRCPC, ${ }^{*}$ Richard Bachand Pharma D, $†$ Laurie Ainsworth MSC

Purpose: To evaluate the effectiveness of short-acting anesthetic drugs and techniques to achieve recovery room bypass criteria after minor surgery in a community hospital environment.

Methods: After agreement by a multidisciplinary committee, a pilot project was undertaken to assess the usefulness of ultra- short acting anesthetic drugs and pre-emptive analgesia to facilitate rapid recovery from general anesthesia. A cohort of I 00 ASA I-II patients aged 18-65 yr undergoing simple knee arthroscopy or minor peripheral orthopedic procedures was compared to a similar cohort treated in the three months prior to the study period. Outcomes of interest included patient morbidity, success in achieving post-anesthesia care unit (PACU) bypass criteria, impact upon nursing resources, duration of operating room (OR) and hospital stay, and pharmaceutical costs before and after implementation.

Results: No patient morbidity was demonstrated prior to discharge home, and successful PACU bypass occurred in $83 \%$ of cases. Achievement of PACU discharge criteria while in the OR did not prolong the OR time, and discharge from hospital occurred earlier in the patients who did not require PACU care $(P=0.0006$ all "fast-track cases" vs all "controls"). Nursing complaints were more numerous when the day surgery personnel did not normally participate in PACU care. The cost of anesthetic care was signifcantly more using ultra-short acting drugs (CDN \$14.17 vs CDN $\$ 20.57$ ), but closer adherence to protocol could reduce this differential (CDN \$18.84).

Conclusion: Not all patients who receive a general anesthetic require admission to a phase I recovery facility. However, the justification for use of more expensive pharmaceuticals to achieve PACU bypass requires extensive changes in operating systems and voluntary professional behaviours.
Objectif : Évaluer l'efficacité d'anesthésiques et de techniques à action brève permettant d'éviter le séjour en salle de réveil (SR) après une intervention mineure dans un hôpital communautaire.

Méthode : Ayant reçu l'accord d'un comité multidisciplinaire, on a mené un projet pilote pour évaluer l'utilité d'anesthésiques à action très brève et d'analgésie préventive pour faciliter une récupération rapide de l'anesthésie générale. Une cohorte de 100 patients d'état physique I-II, de 18-65 ans, devant subir une arthroscopie simple du genou ou une intervention orthopédique périphérique mineure, a été comparée à une cohorte semblable traitée pendant les trois mois qui ont précédé l'étude. Les paramètres étudiés comprennent la morbidité, la possibilité de satisfaire aux critères permettant d'éviter la salle de réveil, les conséquences sur les effectifs infirmiers, la durée du séjour en salle d'opération (SO) et à l'hôpital et la différence de coût des médicaments avant et après l'application du protocole.

Résultats : Aucune morbidité n'a été démontrée avant le départ du patient de l'hôpital et le fait d'éviter la SR a été possible dans $83 \%$ des cas. L'atteinte des critères permettant d'accorder le congé de la SR alors même que le patient est encore en SO n'a pas entraîné de prolongation du temps en SO et la sortie de l'hôpital a été plus hâtive chez les patients qui n'ont pas eu besoin de soins de $S R(P=0,0006$ - tous les cas sous "protocole accéléré" vs tous les "témoins"). Les plaintes du personnel infirmier ont été plus nombreuses lorsque le personnel de chirurgie de jour ne participait pas normalement aux soins de la SR. Le coût des anesthésiques a été un peu plus élevé avec l'emploi de médicaments à action très brève (14,17 \$CAN vs 20,57 \$ CAN), mais une adhésion plus ferme au protocole pouvait réduire cette différence (18,84 \$CAN).

Conclusion : Nul n'est besoin d'admettre, dans une unité de réveil de phase I, tous les patients ayant subi une anesthésie générale. Cependant, la justification de l'usage de médicaments plus chers qui permettent d'éviter la SR nécessite des changements importants d'organisation des interventions et de la collaboration professionnelle volontaire.

From the Departments of Anesthesiology* and Phamacy, † Capital Health Region, Victoria, and the Department of Statistics, $\ddagger$ Simon Fraser University, Vancouver, British Columbia, Canada.

Address correspondence to: Dr. Peter G. Duncan, Department of Anesthesiology, Victoria General Hospital, 35 Helmcken Road,

Victoria, British Columbia V8Z 6R5, Canada. Phone: 250-727-4152; Fax: 250-727-4439; E-mail: pgd@home.com

Acknowledgement: This study was supported in part by an unrestricted grant from Baxter Pharmaceuticals (Canada).

Accepted for publication January 5, 2001.

Revision accepted February 5, 2001. 
I $\mathrm{N}$ recent years the need for efficiency in the operation of surgical units has led to various initiatives, one of which is the elimination of the mandatory post-anesthesia care unit (PACU) stay. This is achieved by the use of new, rapidly-eliminated sedatives and analgesics, facilitated pain management using non-opioids and local anesthetics, and minimally invasive surgical techniques. The essence of the process is that the patient will achieve the state of recovery associated with safe discharge from the PACU even before arrival, and therefore can be transferred directly from the operating room (OR) to the next level of care. ${ }^{1}$

While accelerated (or "fast-tracked") care may seem desirable, it is usually achieved at the increased expense associated with new pharmaceuticals. ${ }^{2}$ To recapture the savings associated with PACU bypass, the liberated human resources of that unit must either be eliminated, or alternatively reassigned in a beneficial fashion. Failing that, the savings associated with expedited patient care plans are mere fiction, and cannot be used to offset the increased drug costs.

Much of the work to improve OR effectiveness has occurred in academic medical centres, ${ }^{3}$ and widespread application of the techniques to community clinical practice is unsubstantiated. Community practices tend to run more efficiently, for the time-cost of education does not enter into the schedule. The relative constancy of the personnel (surgical, anesthetic, and nursing) allows for familiarity and speed, in contrast to the changes associated with various residents and students becoming involved in the case. On the other hand, long-standing community practices may be difficult to modify without clear demonstration of benefit. In addition, the non-uniform educational background of the various participants may lead to variable knowledge of new drugs and procedures.

The objective of this study was to determine if a "fast-track" protocol would lead to cost-effective improvement in how a community hospital handled its case-load. We limited our study group to patients undergoing a suitable common procedure, and evaluated the outcomes using traditional measures of cost and efficiency.

\section{Methods}

A multidisciplinary fast-track steering group composed of representatives of anesthesia, nursing, and pharmacy was struck to oversee the planning and implementation of the study. Following institutional approval, the department of anesthesia of the Capital Health Region initiated a limited program of elective PACU bypass in two primary care hospitals in November, 1999.
Although these two acute care sites function under a single management, they each present a different PACU format: in the one hospital (B) the day surgical area and staff are distinct from those of the phase I recovery area, while the other hospital (A) has an integrated nursing unit in a single physical location for both phase I and II recovery. Anesthetic and surgical staff worked principally in one hospital or the other, and nursing staff did not move between units.

Patients to be fast-tracked were limited to those between the ages of 18 and $60 \mathrm{yr}$, with no significant medical illness (ASA I or II), and scheduled to undergo knee arthroscopy or other minor orthopedic surgery on an outpatient basis. It was felt that 100 consecutive cases ( 50 from each site) would be sufficient to demonstrate the safety and effectiveness of the procedure. These 100 cases would be compared to 100 cases similar in terms of age and procedure. These comparison patients were drawn consecutively from the records of each hospital beginning three months prior to the study's inception.

The nursing staffs of the OR and recovery areas were in-serviced on the initiative, and criteria for bypass were adopted as published in the literature. ${ }^{1}$ These are the standard Aldrete criteria, plus control of pain and emetic symptoms. The anesthetic staff was informed of the project at several staff meetings, and were encouraged to meet the objective of having at least $50 \%$ of their eligible patients meeting the bypass criteria. Anesthesiologists were provided information about the costs of the drugs, and instructed as to the nursing staff requirements (advance warning of each patient's destination, effective control of pain and emetic symptoms, and removal of $i v$ lines). The anesthesiologist also had to have no expectation of immediate reception by the nursing staff as the patient entered the phase II surgical unit. The anesthesiologists were not, however, given a specific protocol to follow, but only told (and reminded each morning prior to beginning their list) of the objective of accelerated recovery they were expected to meet.

Prior to the study, the pharmacy did not have remifentanil available, and desflurane vaporizers were not present at all anesthetizing sites. However, all anethesiologists were accustomed to using desflurane in other clinical situations and, at the inception of the study, both these drugs were made available for all study patients, as were instructional materials about their use. ${ }^{4}$ To assist the attending anesthesiologist, one of the study authors contacted the assigned anesthesiologist prior to each daily list to ensure comfort with how the newer drugs (if chosen) were to be used. The use of multi-modal pre-emptive analgesia was encour- 
TABLE I Post-operative needs of the patients expected to bypass the post-anesthesia care unit (PACU) ("cases") or the historic "control" subjects

\begin{tabular}{lllll}
\hline & $\begin{array}{l}\text { Hospital A } \\
\text { cases: } \\
(n=49)\end{array}$ & $\begin{array}{l}\text { Hospital A } \\
\text { controls: } \\
(n=51)\end{array}$ & $\begin{array}{l}\text { Hospital B } \\
\text { cases: } \\
(n=50)\end{array}$ & $\begin{array}{l}\text { Hospital B } \\
\text { controls: } \\
(n=51)\end{array}$ \\
\hline Pain >T3's & 7 & 28 & 5 & 15 \\
PONV & 3 & 5 & 7 & 4 \\
Other & - & - & 5 & 2 \\
\hline
\end{tabular}

Pain $>$ T3's=pain requiring therapy with analgesics stronger than acetaminophen with codeine $30 \mathrm{mg}$.

aged. There was, however, no obligation to follow a given protocol.

For several years the OR circulating nurses had been entering defined times into the patient health record (entry to the OR, anesthetic "start" and "ready" times, surgical "start" and "finish" times, and time out of the OR). Similar data were recorded in the PACU and the Day Surgical Unit, with definitions used as to when the patient moved from phase I to phase II recovery, when he or she was fit to go home, and when actual departure occurred. These points were extracted from the record of all study and control cases, transferred to a spreadsheet, and interval times calculated.

The anesthetic and recovery records were examined for specific details as to drugs administered, the disposition of the patient after the OR procedure, and any specific treatment required: pain relief, control of postoperative nausea and vomiting (PONV), etc.. Failure of bypass was noted, and the cause attributed to the anesthetic, the surgical procedure, or the medical status of the patient. The post-operative need for significant medical therapy of pain (defined as a need greater than acetaminophen with codeine), PONV, or of any other complaint was noted. Finally, system problems noted by the day surgical area were documented by the staff, and reviewed by the fast track study group.

Costs for the peri-operative anesthetic and recovery drugs used were calculated from each patient record by the pharmacy department using known hospital acquisition prices. The cost of volatile anesthetic gases was calculated using the method of Dion. ${ }^{5}$ The costs of nitrous oxide $\left(\mathrm{N}_{2} \mathrm{O}\right)$ and oxygen $\left(\mathrm{O}_{2}\right)$ were not included. Following a survey of the departmental staff, the usual fresh gas flows for isoflurane and desflurane were set at $\left.2 / 1\left(\mathrm{~N}_{2} \mathrm{O}\right) / \mathrm{O}_{2}\right)$ and $0.5 / 0.5 \mathrm{lpm}$ respectively. Remifentanil infusion therapy was calculated on a loading dose of $0.5 \mu \mathrm{g} \cdot \mathrm{kg}^{-1}$, followed by an infusion of $0.375 \mu \mathrm{g} \cdot \mathrm{kg}^{-1} \cdot \mathrm{min}^{-1}$ for the duration of the anesthetic.
No adjustment was made for drug wastage.

Two-way analysis of variance was used to test for hospital and study group differences in duration of surgery, total OR time, time to discharge, and total hospital stay. The impact of adjusting for surgeon and anesthesiologist (those contributing more than ten patients to the data base) was assessed. Tests of association between surgeon/anesthesiologist and bypass rates for cases were done using Fisher's exact test. SPSS 7.5 and Splus 4.5 were used to perform the analyses. A $P$ value of 0.05 was used for all tests. It should be noted that we tested multiple hypotheses so that our overall study error rate may be larger than 0.05 .

Results

The overall success rate of the fast-track project was $83 \%$, with only $17 / 99$ patients failing to achieve "PACU bypass criteria" upon discharge from the OR. The reasons for failure were: medical indications for monitoring in the PACU (eight patients), failure of the anesthetic to achieve PACU discharge criteria (five patients), and change from the scheduled surgical procedure (four patients). One patient opted to be done under monitored anesthetic care, reducing the assessed number of cases to 99 . The patients who received a "fast-track" anesthetic required less analgesia in the recovery facility than did the control subjects, and had a similar need for symptomatic control of PONV (Table I). One patient in the bypass group required specific therapy for an acute panic reaction.

Analysis of the time associated with patient care (Table II) shows that the average time in the OR was not significantly prolonged by the need to achieve suitable recovery. Moreover, the time for the surgical procedure was not increased by the expected difficulty associated with patient movement during unduly "light anesthesia"; patient movements or signs of awareness were not observed in any instance. As expected, the study patients as a group experienced a shorter total hospital stay $\left(\mathrm{F}_{1,197}=16.7, P=0.00006\right)$, a benefit more marked when only the "successful" fast-track patients were compared to all those ("controls" and failed bypass subjects) who visited the PACU. The average stay in the phase I recovery area was over one hour in the historical controls; the stay for those study subjects who failed to bypass PACU was even greater $(75 \mathrm{~min})$.

There was a significant interaction between the hospital and group effects on duration of surgery $\left(\mathrm{F}_{1,197}=6.8, P=0.01\right)$ : hospital " $\mathrm{A}$ " had shorter durations for surgery for both the cases and the controls. Hospital "B" had longer durations than hospital "A", especially for the controls. Once the influence of which surgeon operated (of those with ten or more 
TABLE II The mean times (in minutes \pm SD) for various activities during the hospital stay

\begin{tabular}{lllll}
\hline & Cases $(n=99)$ & Controls $(n=102)$ & Bypass $(n=82)$ & PACU $(n=119)$ \\
\hline From arrival in OR to start of surgery & $13.5 \pm 5.2$ & $13.1 \pm 4.9$ & $13.5 \pm 5.3$ & $13.2 \pm 4.9$ \\
From surgery start to surgery finish & $25.6 \pm 11.3$ & $28.4 \pm 15.52$ & $24.5 \pm 9.8$ & $28.7 \pm 15.6$ \\
From surgery finish to exit from OR & $7.5 \pm 3.5$ & $5.1 \pm 3.3$ & $7.5 \pm 3.6$ & $5.4 \pm 3.5$ \\
Total time in OR & $46.6 \pm 10.7$ & $46.5 \pm 14.6$ & $45.4 \pm 9.3$ & $47.3 \pm 14.7$ \\
Total time in PACU & \multicolumn{1}{c}{} & $61.6 \pm 25.1$ & $51.6 \pm 28.4$ & $74.5 \pm 31.5$ \\
From exit from OR to ready for home & $61.9 \pm 40.6$ & $87.3 \pm 27.3$ & $24.7 \pm 29.4$ & $22.5 \pm 19.9$ \\
From ready for home to actual discharge & $23.9 \pm 28.0$ & $22.9 \pm 20.0$ & $121.8 \pm 42.8$ & $160 \pm 36.7$ \\
Total Stay & $132.4 \pm 49.3$ & $156.8 \pm 33.4$ & \\
\hline
\end{tabular}

"Cases" refers to patients eligible for PACU bypass; "Controls" refers to historic matched subjects. "Bypass" refers to subjects who successfully avoided any stay in PACU; "PACU" refers to the total number of subjects (including controls and fast-track) who required Phase I admission.

TABLE III The cost of anesthetics used in the post-anesthesia care unit "(PACU) bypass" and "control" cases

\begin{tabular}{|c|c|c|}
\hline & $\begin{array}{l}\text { Fast-track cases } \\
(n=99)\end{array}$ & $\begin{array}{l}\text { Control cases } \\
(n=102)\end{array}$ \\
\hline NSAID premed (oral) & $\$ 0.50(n=90)$ & - \\
\hline NSAID in OR (parental) & - & $\$ 1.78(n=43)$ \\
\hline Propofol (induction) & $\$ 3.87$ & $\$ 3.87$ \\
\hline Propofol infusion & & $\$ 0.32(n=4)$ \\
\hline Narcotics in OR & $\$ 8.44$ & $\$ 0.75$ \\
\hline Volatile anesthetic & $\$ 1.50$ & $\$ 1.27$ \\
\hline Antiemetic in OR & $\$ 0.10(n=20)$ & $\$ 0.19(n=28)$ \\
\hline Sedative (midazolam) & $\$ 0.17(n=48)$ & $\$ 0.43(n=58)$ \\
\hline Neuromuscular blocker & $\$ 2.00(n=30)$ & $\$ 0.89(n=13)$ \\
\hline Reversal agent & $\overline{-}$ & $\$ 0.30(n=8)$ \\
\hline Bupivacaine $0.5 \%$ with epi. & $\$ 3.88$ & $\$ 3.88$ \\
\hline Post-op analgesics (doses) & $\$ 0.03(n=34)$ & $\$ 0.39(n=109)$ \\
\hline Post-op antiemetics (doses) & $\$ 0.08(n=3)$ & $\$ 0.10(n=5)$ \\
\hline Total costs per case & $\$ 20.57$ & $\$ 14.17$ \\
\hline
\end{tabular}

The total cost of pharmaceuticals used in each group was totalled, then normalised to an individual case. The calculation of volatile and infusion drug consumption is explained in the text; all calcula tions assume a $70 \mathrm{~kg}$ weight, and a $30 \mathrm{~min}$ anesthetic time. Where " $n$ " is not stated, all patients in the group received the medication noted.

TABLE IV Projected costs of an average fast-track case of a 70 $\mathrm{kg}$ individual undergoing a 30- min procedure; assumes neither paralysis nor rescue antiemetics are needed

\begin{tabular}{ll}
\hline & Cost $(C D N \$)$ \\
\hline NSAID (naproxen $500 \mathrm{mg} \mathrm{po})$ & $\$ 0.20$ \\
Remifentanil $\left(0.5 \mathrm{ug} \cdot \mathrm{kg}^{-1}\right.$ load, $\left.0.375 \mu \mathrm{g} \cdot \mathrm{kg}^{-1} \cdot \mathrm{min}^{-1}\right)$ & $\$ 8.43$ \\
Desflurane $(1.5 \%$ @low FGF) & $\$ 1.50$ \\
Propofol $\left(2 \mathrm{mg} \cdot \mathrm{kg}^{-1}\right)$ induction & $\$ 3.87$ \\
Antiemetic (droperidol $500 \mu \mathrm{\mu g})$ & $\$ 0.35$ \\
Sedative (midazolam l mg) & $\$ 0.55$ \\
Bupivacaine $0.5 \%$ with epinephrine $(20 \mathrm{~mL})$ & $\$ 3.88$ \\
Analgesic (acetaminophen $500 \mathrm{mg}$ & $\$ 0.06$ \\
with codeine $30 \mathrm{mg})$ x 2 tablets & \\
Total & $\$ 18.84$ \\
\hline
\end{tabular}

cases) was controlled in the statistical model, the hospital main effect was not significant to the duration of the OR. That is, the effect of hospital of service on the OR duration is probably due to the difference between surgeons, and not the process of care.

The nursing staff raised a few concerns during the study period. These included the observations that the patients arrived cold, that they arrived during busy periods, and that bypass criteria were not actually being met. Noteworthy is that these concerns were expressed only in the hospital where the two phases of the recovery process are physically distinct. Finally, it was irritating to nursing in both hospitals that the patients were ready to go home earlier, yet discharge was delayed for a variety of system reasons.

Success at achieving PACU bypass was not dependent upon who provided the clinical services: by Fisher's exact test surgeons or anesthesiologists (with more than five patients each) and percentage bypass were not significantly related. While there was quite a range of success rates, the study had limited power to detect a difference in the bypass success rate and individual members of the surgical or anesthetic staff.

The direct drug costs associated with the "fast-track" procedure ( $\$ 20.57$ per case) were greater than those associated with the "control" anesthetics (\$14.17; Table III). The calculated cost of the recommended protocol for fast-tracked cases (Table IV; \$18.84) suggests that there were further cost savings to be made if compliance with the guidelines had been complete.

\section{Discussion}

The need to control costs in anesthetic practice has become commonplace, particularly in academic departments. ${ }^{6}$ As newer pharmaceutical agents come to market there is pressure to adopt them into clinical practice, arguing that the cost of their engineered advantages will be offset by fiscal gains in other areas. However, this has 
not been easy to demonstrate. For example Rose ${ }^{7}$ could not demonstrate that more expensive anesthetic drugs provided significant gains in terms of recovery outcomes after hysterectomy, save in the incidence of emetic symptoms. A basic challenge with new drug introduction is to show that the "efficacy" suggested from controlled clinical trials can be translated into "effectiveness" in routine clinical practice.

We were able to successfully demonstrate, as have others, ${ }^{1}$ that following selected procedures not all patients need to go to a phase I recovery area after receiving a general anesthetic. In our study over $80 \%$ of patients were successfully fast-tracked, no patient morbidity occurred, and no impact on the time spent in the OR was demonstrable. Since PACU costs usually run approximately $1 / 10$ of those of the OR, ${ }^{8,9}$ any delay in exit from the latter would lose any advantage gained by a PACU-bypass protocol. As motivation to explore the bypass option, the current restriction on recovery nursing resources in the study institution has commonly resulted in delays in admission to the PACU from the OR, adding overtime expenses to scheduled surgical lists. We conclude that a potential solution is to revise PACU protocols to allow for accelerated passthrough or complete bypass by appropriate cases, as is gaining popularity as hospitals reduce their mandatory recovery periods. ${ }^{10}$ Important elements for the success of such endeavours include multi-modal pre-emptive analgesia, minimal physiologic trespass, and utilization of pharmaceuticals that lack residual effect beyond that necessary for the procedure.

Since most patients coming to surgery on a day surgical basis are identifiable in advance only by age and scheduled procedure, it is not always possible to ascertain which ones will be medically suitable for a fasttrack protocol. Without such triage an institution cannot reassign recovery resources to harvest the maximum benefit of new anesthetic drugs, and the potential for recovery of direct drug costs is reduced. However, we found that not all failures of the system pertain to unexpected medical illness in the patients. It was of interest that the two hospital day-surgical nursing staffs experienced different satisfaction with the procedure of PACU bypass, depending on whether the Phase II staff were familiar with the usual symptoms experienced by patients coming out of an operating suite. This dependence upon which nurse provides post-anesthesia observation has been demonstrated earlier by Pavlin et al. ${ }^{11}$ The further delay in patient discharge due to "system" problems was also demonstrated by Waddle et al., ${ }^{12}$ and implies that the complex environment of an operating suite requires considerable engineering to gain the theoretical advantages of new pharmaceuticals.

While success in meeting bypass criteria was not statistically related to which physicians provided care, the study had a low power to detect such differences. However, there was a large range in success rates, and compliance with the suggested anesthetic guidelines for fast-tracking was not uniform. The study protocol was constructed to avoid the antagonism of private practitioners by not being excessively prescriptive, and only offered them information, reminders, and the opportunity to experience practice with newer drugs. Their successful adherence to protocol would also offer the reward of less frustration with the daily OR congestion from a PACU backlog. On the negative side, it can be speculated that, in a fee-for-time payment schedule, delays in the OR are still compensated. Alternately, the physicians may have been unfamiliar with aspects of care important to successful fast-track anesthetics. For example, some anesthesiologists are reluctant to ventilate patients with positivepressure while using a laryngeal mask airway, while others perceive a need to use paralysis to introduce artificial airways or to avoid chest wall rigidity with narcotics. Finally, it may be that some are simply not interested in changing their practice, perhaps due to perceptions that financial concerns should not determine how they practice medicine. Whatever the reason for variable success rates, it suggests that voluntary programs of care mapping are unlikely to be totally successful in achieving uniform clinical objectives.

In an early assessment of physician behaviour it was pointed out that providing up-to-date information, making group decisions, and having rewards for successful behaviour are important factors determining what physicians do. ${ }^{13}$ In anesthesia practice, it has not been possible to sustain economic advantages with voluntary practice guidelines, ${ }^{14,15}$ although Berman ${ }^{16}$ was able to transiently reduce the use of technology (warming blankets) by anesthesia residents with a cost feedback system. The use of a more detailed and comprehensive peri-operative clinical pathway ${ }^{17}$ has been shown to save OR resources in a more predictable fashion. Perhaps this is due to the greater peer pressure placed upon individual physicians by the multiple participants working together (surgeons, anesthesiologists, nurses, and administrators) to adhere to the recommended pattern of practice. In a most interesting article related to physicians' compliance with practice guidelines Posner et al. ${ }^{18}$ assessed the response of cardiac surgeons and anesthesiologists to a necessary change in practice management to meet economic objectives. In spite of the potential for both personal and collective financial gain, these physicians were 
unwilling to give up their perceived autonomy in patient care decisions in the face of the "threat" of bureaucratic guidelines (even those generated by their own consensus), resulting in failure of the cost-saving initiative. It is impossible to know whether similar emotions were operative in the present study.

Limitations of the study

Definitive proof in a biologic system demands control of as many variables as possible. To do this in medicine requires a randomized study design; unfortunately, this is not always possible in clinical studies. The alternative is to observe the outcomes of clinical practice, and the change that occurs over time. While the conclusions must be tempered, ${ }^{19}$ the observations can provide valuable insight. The current study used relatively consistent anesthesia, surgical, and nursing staffs, and compressed the study into as brief a period as possible. As mentioned, the study design and case numbers did not allow differentiation between hospital effects and the effects of individual surgeons and anesthesiologists.

The charting of OR times was done not by a dedicated research person, but was part of an established clinical audit program. The system was well understood, with few mistakes anticipated. Individual patient expenses were not calculated, for the accuracy of recording in a clinical practice would be open to question; the normalization of group data to a single "case" was a reasonable approximation. There was no specific follow-up of these patients by anesthesia once they met discharge criteria from the hospital, but few late complications are expected for this type of procedure, and private practitioners tend to hear quickly from their surgical colleagues if patients experience complications. Finally, we chose to study a single procedure rather than a wider patient population, taking advantage of the standardisation of the surgical trauma and the ease with which post-operative pain could be controlled by multimodal therapy.

\section{Conclusion}

We conclude that not all patients who receive a general anesthetic need to go to a phase I recovery facility. However, to create an efficient system that justifies the more expensive pharmaceuticals that facilitate PACU bypass, extensive changes in operating systems and professional behaviours will be necessary.

\section{References}

1 White PF, Song D. New criteria for fast-tracking after outpatient anesthesia: a comparison with the modified Aldrete's scoring system. Anesth Analg 1999; 88: 1069-72.
2 Suttner S, Boldt J, Schmidt C, Piper S, Kumle B Cost analysis of target-controlled infusion-based anesthesia compared with standard anesthetic regimens. Anesth Analg 1999; 88: 77-82.

3 Overdyk FJ, Harvey SC, Fishman RL, Shippey F. Successful strategies for improving operating room efficiency at academic institutions. Anesth Analg 1998; 86: 896-906.

4 Reves JG. Educational considerations for the clinical introduction and use of remifentanil. Anesth Analg 1999; 89: S4-6.

5 Dion P. The cost of anaesthetic vapours (Letter). Can J Anaesth 1992; 39: 633.

6 Valenzuela RC, Johnstone RE. Cost containment in anesthesiology: a survey of department activities. J Clin Anesthesia 1997; 9: 93-6.

7 Rose K, Cohen MM. Are anesthetic drug costs related to patient outcome? Anesthesiology 1996; 85: A937 (abstract).

8 Macario A, Vitez TS, Dunn B, McDonald T. Where are the costs in perioperative care? Analysis of hospital costs and charges for inpatient surgical care. Anesthesiology 1995; 83: 1138-44.

9 Johnstone RE, Evans K, Hosaflook C Costs per minute for an operating room. Anesthesiology 1999; 91: A1225 (abstract).

10 Mamaril M. ASPAN position statement. Fast-tracking the postanesthesia patient: the pros and cons. J Perianesth Nurs 2000; 15: 89-93.

11 Pavlin DJ, Rapp SE, Polissar NL, Malmgren JA, Koerschgen $M$, Keyes $H$. Factors affecting discharge time in adult outpatients. Anesth Analg 1998; 87: 816-26.

12 Waddle JP, Evers AS, Piccirillo JF. Postanesthesia care unit length of stay: quantifying and assessing dependent factors. Anesth Analg 1998; 87: 628-33.

13 Harris JS. Why doctors do what they do: determinants of physician behavior. J Occup Med 1990; 32: 1207-11.

14 Kirsch MA, Carrithers JA, Hagan RH, Borra HM. Effects of a low-cost protocol on outcome and cost in a group practice setting. J Clin Anesth 1998; 10: 416-24.

15 Lagasse RS, Jiang HJ, Ciccone K, et al. Effect of practice guideline dissemination strategies on physician behavior and patient outcomes. Anesthesiology 1998; 89: Al331 (abstract).

16 Berman MF, Simon AE. The effect of a drug and supply cost feedback system on the use of intraoperative resources by anesthesiologists. Anesth Analg 1998; 86: 510-5.

17 Macario A, Horne M, Goodman S, et al. The effect of a perioperative clinical pathway for knee replacement surgery on hospital costs. Anesth Analg 1998; 86: 978-84. 
18 Posner KL, Gild WM, Winans EV. Changes in clinical practice in response to reductions in reimbursement: physician autonomy and resistance to bureaucratization. Med Anthropology Quarterly 1995; 9: 476-92.

19 Duncan PG That was then, this is now! The value of observing change. Anesth Analg 1998; 86: 225-7. 